\title{
Evaluating Sphingosine and its Analogues as Potential Alternatives for Aggressive Lymphoma Treatment
}

\author{
Constantin Bode ${ }^{a}$ Max Berlin ${ }^{a}$ Franziska Röstela, ${ }^{a, b} \quad$ Bianca Teichmann ${ }^{a}$ \\ Markus H. Gräler \\ ${ }^{a}$ Molecular Cancer Research Centre, Charité University Medical School, Berlin; ${ }^{b}$ Department of \\ Anesthesiology and Intensive Care Medicine, Center for Sepsis Control and Care (CSCC), and the \\ Center for Molecular Biomedicine (CMB), University Hospital Jena, Jena, Germany
}

\section{Key Words}

Sphingosine - Ceramide - Autophagy $\cdot$ Apoptosis - Protein kinase $C \cdot$ Diffuse large B cell lymphoma

\begin{abstract}
Background: Ceramide (Cer) and sphingosine (Sph) interfere with critical cellular functions relevant for cancer progression and cell survival. While Cer has already been investigated as a potential drug target for lymphoma treatment, information about the potency of sphingosine is scarce. The aim of this study therefore was to evaluate Sph and its synthetic stereoisomer L-threo-sphingosine (Lt-Sph) as potential treatment options for aggressive lymphomas. Methods: Diffuse large B cell lymphoma (DLBCL) cell lines were incubated with Sph and LtSph and consequently analysed by flow cytometry (FACS), enzyme-linked immunosorbent assay (ELISA), liquid chromatography coupled to triple-quadrupole mass spectrometry (LC/ MS/MS), electron microscopy, and Western blot. Results: Sph induced cell death and blocked cell growth independently of S1P receptors in different DLBCL cell lines. Three different modes of Sph-mediated cell death were observed: Apoptosis, autophagy, and protein kinase C (PKC) inhibition. Generation of pro-apoptotic Cer accounted only for a minor portion of the apoptotic rate. Conclusion: Sph and its analogues could evolve as alternative treatment options for aggressive lymphomas via PKC inhibition, apoptosis, and autophagy. These physiological responses induced by different intracellular signalling cascades (phosphorylation of JNK, PARP cleavage, LC3-II accumulation) identify Sph and analogues as potent cell death inducing agents.
\end{abstract}




\section{Introduction}

DLBCL is the most common of the aggressive lymphomas with estimated 70.000 new cases and 19.000 deaths in the United States in 2013 [1]. It is characterized by highly heterogenous morphology, biology, and clinical presentation [2]. Different patterns of gene expression give rise to 2 distinct subtypes of DLBCL: Germinal center-like (GCB) and activated $B$ cell-like (ABC) [3]. A major characteristic of ABC DLBCL is the constitutive activation of the nuclear factor-kappa B (NF-кB) pathway [4]. A majority of GCB DLBCL was found to be dependent on the phosphatidylinositide 3-kinase (PI3K) and protein kinase B (Akt) pathway [5]. In contrast to slow-growing indolent non-Hodgkin's lymphomas (NHL), rapidly growing DLBCL is often curable with a success rate greater than 50\% [1]. ABC DLBCL has the worst prognosis with 3-year overall survival rates of around 40\% [6].

Sphingolipids like Sph, sphingosine 1-phosphate (S1P), and Cer are important determinants for cell fate $[7,8]$. While S1P signalling through G protein-coupled cell surface S1P receptors is considered as a pro-survival factor [9], Cer and Sph both induce cell death, albeit using different signalling pathways $[7,8]$. The exact mechanisms of both lipids are not completely understood (reviewed in $[10,11]$ ) . Cer for example interferes with mitochondrial functions $[12,13]$, but also upregulates apoptosis-inducing proteins like Bcl$\mathrm{xS}$ and caspase-9 [14]. It directly activates PKC-zeta and binds to cathepsin D to support its proteolytic maturation and activation $[15,16]$. Cer also induces autophagy. Dependent on the cell system used, the mechanism involves the induction of ER stress [17], suppression of Akt [18], activation of JNK [19], up-regulation of Beclin 1 [20], and expression of the mitochondrial BH3-only protein BNIP3 [21]. Little is known about the signalling pathways that transmit Sph-induced apoptosis. It inhibits PKC and mitogen-activated protein kinase (MAPK) [22, 23], but may also act through ceramide synthase-dependent conversion to Cer [24]. It can be rapidly phosphorylated by sphingosine kinases (SphK) type 1 and 2 to S1P $[25,26]$ which has antiapoptotic functions predominantly by activating S1P receptors $[9,27]$. These two different metabolic conversions may explain antipodal observations demonstrating downor upregulation of antiapoptotic Bcl-2 proteins in different cell systems [28, 29].

The generation of pro-survival S1P by SphKs supports tumor growth (reviewed in [30]). Current strategies for cancer treatment therefore include inhibition of SphKs, particularly SphK1, as a potentially new therapeutic avenue [31]. While prevention of S1P generation is generally regarded as the main anti-cancer effect due to abrogation of pro-survival S1P signalling, the effect of concomitant Sph accumulation has typically not been considered as being effective. Different interventions in sphingolipid metabolism like deficiency of SphK2 [32] or the S1P degrading enzyme S1P-lyase [33] however result in increased Sph concentrations. Moreover sphingosine analogues were tested for their ability to inhibit SphKs [34]. SphK inhibitors that are structurally related to Sph may not only prevent Sph phosphorylation, but could also share functional properties of Sph. We therefore investigated the effect of Sph on different DLBCL in order to better understand the idiosyncratic functions of Sph in the context of aggressive lymphomas, and to explore Sph accumulation and Sph analogues as alternative treatment options for aggressive lymphomas.

\section{Materials and Methods}

\section{Chemicals}

The following chemicals were used throughout the study: S1P (Sigma), Sph (Sigma), Lt-Sph (Avanti Polar Lipids), C17-Sph (Avanti Polar Lipids) sphinganine (Avanti Polar Lipids), C15-Cer (Matreya), C16-Cer (Matreya), phosphatidylserine (PS, Sigma), camptothecin (Sigma), fumonisin B1 (Cayman), 4-deoxypyridoxine (DOP, Sigma).

Cell culture

HT, HBL-1, and U2932 cells were grown in RPMI 1640 (Life Technologies) and OciLy19 in Iscove's modified Dulbecco's medium (IMDM) with 10\% fetal bovine serum (FBS, Biochrom), 1 mM sodium 
pyruvate (PAA Laboratories), 100 units/ml penicillin G (PAA Laboratories), $100 \mu \mathrm{g} / \mathrm{ml}$ streptomycin (PAA Laboratories), and $2 \mathrm{mM}$ L-glutamine (PAA Laboratories) [35].

\section{Analysis of cell growth}

Cells were plated in 96-well plates at 100,000 cells/well and treated with the indicated concentrations of the respective compound. Immediately and 1-4 days later, cells were lysed with CellTiter-Glo luminescent cell viability assay reagent according to the manufacturer's protocol (Promega), and the resulting luminescence was determined with the Victor ${ }^{3}$ plate reader (PerkinElmer).

\section{Determination of apoptosis by FACS}

Cells were plated in 24-well plates at 100,000 cells $/ \mathrm{ml}$ and treated with $3 \mu \mathrm{M}$ (OciLy19) or $5 \mu \mathrm{M}$ (HT, HBL-1, U2932) Sph and Lt-Sph, and with $20 \mu \mathrm{M}$ camptothecin for $4 \mathrm{~h}$. Subsequently cells were washed twice with ice-cold binding buffer (0.01 M Hepes/NaOH (pH 7.4), $0.14 \mathrm{M} \mathrm{NaCl}, 2.5 \mathrm{mM} \mathrm{CaCl} 2)$. Five $\mu$ l fluorescein conjugated annexin V (annexin V-FITC, Immunotools) was added to $100 \mu$ l cell suspension in binding buffer and incubated at room temperature for $15 \mathrm{~min}$. Five $\mu \mathrm{l}$ of $50 \mu \mathrm{M}$ propidium iodide was added immediately before FACS analysis using the FACSCalibur (Becton Dickinson).

\section{Lipid quantification}

Lipid quantification was done as described [36, 37]. Biological samples (1 ml of medium or 10E6 cells) were adjusted to $1 \mathrm{ml}$ sample volume with $1 \mathrm{M} \mathrm{NaCl}$ in $\mathrm{H} 2 \mathrm{O}$ and transferred into a glass centrifuge tube. After addition of $1 \mathrm{ml}$ of methanol and $200 \mathrm{ml}$ of $6 \mathrm{M} \mathrm{HCl}$, the samples were vortexed. Chloroform ( $2 \mathrm{ml}$ ) was added, and the samples were again vigorously vortexed for $2 \mathrm{~min}$. After the samples were centrifuged for 3 $\min$ at $1,900 \mathrm{xg}$, the lower chloroform phase was transferred to another glass centrifuge tube. After the lipid extraction was repeated with $2 \mathrm{ml}$ of chloroform, the chloroform phases were combined and vacuum dried in a speed-vac for $45 \mathrm{~min}$ at $50{ }^{\circ} \mathrm{C}$. The QTrap triple-quadrupole mass spectrometer (ABSciex) interfaced with a Merck-Hitachi Elite LaChrom chromatograph and autosampler was used for electrospray ion (ESI) LC/MS/MS analysis. Positive ion ESI LC/MS/MS analysis was employed for detection of all analytes. The multiple reaction monitoring transitions for the detection were as follows: C17-Sph m/z 286/268, C15Cer m/z 524/264, Sph m/z 300/282, sphinganine m/z 302/284, S1P m/z 380/264, C16-Cer m/z 538/264 C24-Cer m/z 650/264. Liquid chromatographic resolution of all analytes was achieved using a MultoHigh RP 18-3 $\mu$ m column (2 mm x $60 \mathrm{~mm}$, CS Chromatographie Service). The elution protocol was composed of a 9 min column equilibration with $10 \%$ solvent A (methanol) and $90 \%$ solvent B (1\% formic acid) followed by sample injection and a 20 min period with $100 \%$ solvent A. Samples were infused into the ESI source through an electrode tube at a rate of $300 \mu \mathrm{l} / \mathrm{min}$. Standard curves were generated by adding increasing concentrations of the analytes to $300 \mathrm{pmol}$ of C17-Sph and C15-Cer (internal standards). Linearity of the standard curves and correlation coefficients were obtained by linear regression analyses. All mass spectrometry analyses were performed with Analyst 1.4 (ABSciex).

\section{Cell cycle analysis}

Cells were plated in 24-well plates at 100,000 cells/ml and treated with $3 \mu \mathrm{M}$ (OciLy19) or $5 \mu \mathrm{M}$ (HT, HBL-1, U2932) Sph and Lt-Sph in the presence or absence of $15 \mu \mathrm{M}$ PS for 3 days. Cells were harvested and resuspended in $400 \mu \mathrm{l}$ hypotonic lysis buffer $(50 \mu \mathrm{g} / \mathrm{ml}$ propidium iodide in 0.5 phosphate buffered saline (PBS) and $0.1 \%$ Triton X-100). After incubation at room temperature for $1 \mathrm{~h}$, the liberated nuclei were analyzed by FACS with the FACSCalibur (Becton Dickinson).

\section{Staining of acidic vesicular organelles}

Cells were plated in 24-well plates at 100,000 cells $/ \mathrm{ml}$ and treated with $3 \mu \mathrm{M}$ (OciLy19) or $5 \mu \mathrm{M}$ (HT, HBL-1, U2932) Sph and Lt-Sph, and with $10 \mu \mathrm{M}$ camptothecin for 1 day. Subsequently acridine orange was added to the cell suspension at a final concentration of $1 \mu \mathrm{g} / \mathrm{ml}$ for $15 \mathrm{~min}$. Cells were washed twice with ice-cold PBS and analyzed by FACS with the FACSCalibur (Becton Dickinson).

\section{Electron microscopy}

Samples were fixed in 2.5\% glutaraldehyde in $0.1 \mathrm{M}$ cacodylate buffer (pH 7.3) overnight. Samples were washed, postfixed with $2 \%$ osmium tetroxide in $0.1 \mathrm{M}$ cacodylate buffer for two hours, dehydrated 
with graded ethanol solutions, and embedded in Epon (SERVA). Semithin sections were stained with Richardson' stain [38]. Ultrathin sections were stained with uranyl acetat and lead citrate [39]. The samples were analyzed on a transmission electron microscope EM 906 (Zeiss, Oberkochen). Sample preparation was done by Petra Schrade (Electron Microscopy Facility, Charité - University Medical School Berlin, Germany).

\section{PKC activity assay}

PKC activity was tested using the PepTag fluorescent protein kinase assay (Promega) according to the manufacturer's protocol. All PepTag PKC assay reaction components were combined on ice, and PKC activity was assayed in a final volume of $25 \mu \mathrm{l}$ of the following mixture: $5 \mu \mathrm{l}$ of $5 \mathrm{x}$ PKC reaction buffer (100 mM HEPES, pH 7.4, 6.5 mM CaCl2, 5 mM dithiothreitol, 50 mM MgCl2, 5 mM ATP), $5 \mu$ of PepTag C1 peptide (PLSRTLSVAAK, $0.4 \mu \mathrm{g} / \mu \mathrm{l}$ in water), $5 \mu \mathrm{l}$ of freshly sonicated PKC activator solution $(1 \mathrm{mg} / \mathrm{ml}$ PS in water), $1 \mu \mathrm{l}$ of peptide protection solution, $3.75 \mu \mathrm{l}$ of water, $1.25 \mu \mathrm{l}$ of either vehicle (methanol), Sph, or Lt-Sph, and $4 \mu \mathrm{l}$ of supplied PKC $(2.5 \mu \mathrm{g} / \mathrm{ml}$ in PKC dilution buffer containing $100 \mu \mathrm{g} / \mathrm{ml} \mathrm{FBS}$ and 0.05\% Triton X-100. Before adding PKC, the mixture was preincubated at $30{ }^{\circ} \mathrm{C}$ for $2 \mathrm{~min}$. After the addition of PKC, the entire reaction mixture was incubated at $30^{\circ} \mathrm{C}$ for $30 \mathrm{~min}$. The reaction was stopped by incubation at $95{ }^{\circ} \mathrm{C}$ for 10 min. Before loading samples on an agarose gel (0.8\% agarose in 50mMTris-HCl buffer, pH 8.0), $2 \mu \mathrm{l}$ of $80 \%$

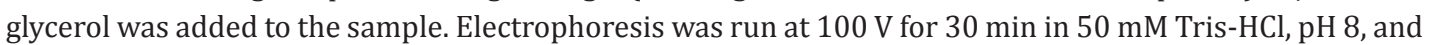
was imaged immediately under UV light. Signals were quantified using ImageJ (NIH).

\section{Quantification of $I L-10$}

Enzyme-linked immunosorbent assay (ELISA) was used to quantify human interleukin-10 (IL-10) in the supernatant of DLBCL cell lines. 300,000 HBL-1 cells/300 $\mu$ l were grown for $24 \mathrm{~h}$ and 60,000 U2932 cells $/ 300 \mu \mathrm{l}$ were grown for $6 \mathrm{~h}$ in 96-well plates (TPP) in the presence and absence of 3-5 $\mu \mathrm{M}$ Sph and Lt-Sph. Subsequently cells were centrifuged at $300 \mathrm{xg}$ and the supernatant was harvested. Maxisorp 96-well plates (NUNC) were coated with the coating antibody provided by the IL-10 ELISA set and processed according to the manufacturer's protocol (Immunotools). Plates were developed with 3,3',5,5'-tetramethylbenzidine (TMB) substrate solution (eBioscience). The reaction was stopped with $1 \mathrm{~N} \mathrm{HCl}$. Absorbance at $450 \mathrm{~nm}$ was detected with the Victor ${ }^{3}$ plate reader (PerkinElmer). Standard curves were generated with 3-300 pg/ml IL-10 and used for quantification.

\section{Determination of AKT phosphorylation and PARP cleavage}

Cells were plated in 6-well plates at 100,000 cells/ml and treated with $3 \mu \mathrm{M}$ (OciLy19) or $5 \mu \mathrm{M}$ (HT, HBL-1, U2932) Sph and Lt-Sph. Subsequently cells were transferred into 96-well plates at a density of 100.000 cells per well and tested for the presence of AKT (protein kinase B), phospho-AKT (pAKT), cleaved poly (ADP ribose) polymerase (PARP), and tubulin with colorimetric in-cell ELISA kits according to the manufacturer's protocol (Pierce Biotechnology). Normalization was performed by whole cell staining with Janus green.

\section{Determination of mTOR and JNK phosphorylation}

Cells were plated in 6-well plates at 100,000 cells/ml and treated with $3 \mu \mathrm{M}$ (OciLy19) or $5 \mu \mathrm{M}$ (HT, HBL-1, U2932) Sph and Lt-Sph. After harvesting they were tested for the presence of the c-Jun N-terminal kinase (JNK), phospho-JNK (pJNK), and Ser2448 phosphorylated mammalian target of rapamycin (mTOR) with ELISA kits according to the manufacturer's protocol (Abcam). Protein concentrations were $130 \mu \mathrm{g} / \mathrm{ml}$ (OciLy19) and $190 \mu \mathrm{g} / \mathrm{ml}$ (HT, HBL-1, U2932) for JNK measurements, $650 \mu \mathrm{g} / \mathrm{ml}$ (OciLy19) and $950 \mu \mathrm{g} / \mathrm{ml}$ (HT, HBL-1, U2932) for pJNK measurements, and $160 \mu \mathrm{g} / \mathrm{ml}$ (OciLy19) and 450 mg/ml (HT, HBL-1, U2932) for mTOR measurements, respectively.

\section{Western-blot analysis}

Western blots were performed according to standard protocols. Cells were plated in 6-well plates at 100,000 cells $/ \mathrm{ml}$ and treated with $3 \mu \mathrm{M}$ (OciLy19) or $5 \mu \mathrm{M}$ (HT, HBL-1, U2932) Sph and Lt-Sph. After harvesting, 200.000 cells were lysed in $20 \mathrm{mM}$ Tris-HCl, pH 7.4, $150 \mathrm{mM} \mathrm{NaCl,} 1 \mathrm{mM}$ EDTA, pH 8.0, 1\% Triton-X-100, $20 \mathrm{mM} \mathrm{NaF}, 0.1 \mathrm{mM} \mathrm{Na}_{3} \mathrm{VO}_{4}$, and complete protease inhibitor cocktail (Roche Applied Science). Lysates (12 - $23 \mu \mathrm{g}$ ) were subjected to 8-16\% Bis-Tris gels (GE Healthcare) according to the manufacturer's 
protocol, and proteins were transferred to Hybond-P polyvinylidene difluoride (PVDF) membranes (GE Healthcare) by wet blotting. Membranes were subsequently blocked with $5 \%$ SlimFast chocolate powder (Allpharm-Vertriebs-GmbH) in Tris-buffered saline and probed with 1:1000 dilutions of the following primary antibodies (Cell Signaling Technology) overnight at $4{ }^{\circ} \mathrm{C}$ : Rabbit anti-LC3A (clone D50G8), rabbit anti-calnexin (clone C5C9), rabbit anti-Ero1-L $\alpha$, or rabbit anti-IRE1 $\alpha$ (clone 14C10). After incubation with a specific horseradish peroxidase (HRP)-labeled secondary antibody against rabbit (Cell Signaling Technology \#7074, 1:2000 dilution), signals were visualized with the enhanced chemiluminescent detection system (ECL) according to the manufacturer's instructions (GE Healthcare).

Statistical analysis

A two-tailed unpaired Student $t$ test was used to determine the significance of differences $\left({ }^{*} \mathrm{p}<0.05\right.$, ${ }^{* *} \mathrm{p}<0.01$, and $\left.{ }^{* * *} \mathrm{p}<0.001\right)$.

\section{Results}

Sph inhibited cell growth and induced apoptosis in DLBCL cell lines

To investigate the impact of cellular Sph accumulation on lymphoma cell growth, different DLBCL cell lines (OciLy19, HT, HBL-1, U2932) were incubated with increasing concentrations of Sph. Determination of cell growth each day over 4 days revealed concentration-dependent growth inhibition after treatment with Sph in all DLBCL cell lines tested, with HBL-1 cells being most resistant to higher Sph concentrations, which were thus treated with 2 -fold higher sphingolipid concentrations (Fig. 1A). All DLBCL cell lines also responded to Lt-Sph with decreased growth, with HT cells showing a lower impact of Lt-Sph than Sph. Lt-Sph is a stereoisomer of naturally occurring D-erythro-sphingosine (Sph). It can be phosphorylated by cells, but the resulting Lt-S1P does not bind and activate S1P receptors, excluding their role in Lt-Sph-induced growth inhibition [40-42].

To test the onset of apoptosis as one possible reason for the observed growth retardation in examined cells, DLBCL cell lines were treated for $4 \mathrm{~h}$ with Sph and Lt-Sph, and with 20 $\mu \mathrm{M}$ camptothecin as positive control. Camptothecin is a cytotoxic quinoline alkaloid which induces apoptosis by inhibiting the topoisomerase type 1 (topo1) [43]. All cell lines were treated with $5 \mu \mathrm{M}$ Sph and Lt-Sph except OciLy19, which were more sensitive to Sph and Lt-Sph incubation and were therefore treated with $3 \mu \mathrm{M}$ Sph and Lt-Sph. Apoptosis was detected by FACS after staining apoptotic cells with annexin $V$ and dead cells with propidium iodide. All DLBCL cell lines responded with 28-59\% apoptosis to camptothecin after $4 \mathrm{~h}$. Sph also induced a high apoptotic rate of 23-32\%, while Lt-Sph was less efficient with apoptotic rates between 12-22\% (Fig. 1B). U2932 cells did not respond to Lt-Sph treatment with apoptosis at all (Fig. 1B). Most of the propidium iodide and annexin V double-positive cells after Lt-Sph treatment did not pass the annexin $V$ single-positive stage and were therefore not considered as apoptotic cells.

Apoptosis is mainly driven by activation of death proteases known as caspases [44], although caspase-independent apoptosis has also been described [45]. Caspases cleave proteins that are essentially required for cellular function and cell survival. One of these many target proteins of caspases is PARP, and cleaved PARP is commonly used as an indicator for caspase-induced apoptosis [46]. Incubation of DLBCL cell lines with Sph and Lt-Sph as mentioned before induced significant PARP cleavage only in OciLy19 DLBCL predominantly after Sph treatment (Fig. 1C). A significant but less pronounced increase in PARP cleavage was also found after Lt-Sph treatment. No important differences in PARP cleavage were observed in similarly treated HT, HBL-1, and U2932 DLBCL cell lines (Fig. 1C). A related pattern was observed for JNK phosphorylation (Fig. 1D). pJNK was significantly increased only in OciLy19 DLBCL treated with Sph, but not Lt-Sph. Both stimuli did not induce pJNK in HT, HBL-1, or U2932 DLBCL cell lines, which obviously depended on different signalling pathways. 


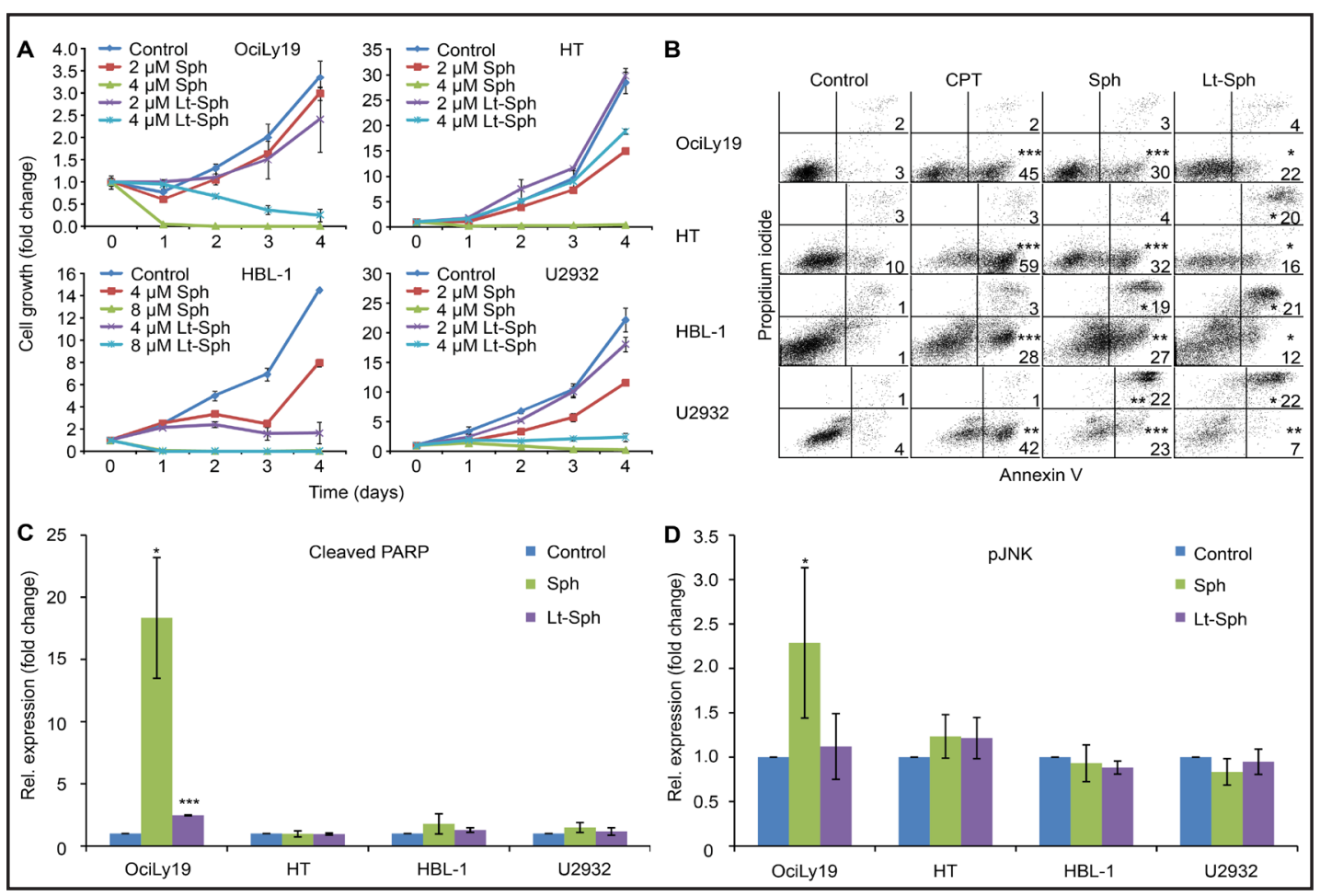

Fig. 1. Impact of Sph and Lt-Sph on cell growth and survival of different DLBCL cell lines (OciLy19, HT, HBL1, and U2932). (A) Analysis of cell growth in the course of 4 days after treatment with indicated concentrations of Sph and Lt-Sph. Shown are means \pm SD, $n=2$. (B) Annexin V and propidium iodide staining of cells $4 \mathrm{~h}$ after treatment with $3 \mu \mathrm{M}$ (OciLy19) and $5 \mu \mathrm{M}$ (HT, HBL-1, U2932) Sph and Lt-Sph, and $20 \mu \mathrm{M}$ camptothecin (CPT). Shown are representative FACS histograms and the percentages of apoptotic (Annexin V positive) and dead (Annexin $\mathrm{V}$ and propidium iodide double-positive) cells of 3 individual experiments, ${ }^{*} \mathrm{p}<0.05$, ${ }^{* *} \mathrm{p}<0.01,{ }^{* * *} \mathrm{p}<0.001$. (C) Analysis of cleaved PARP by colorimetric in-cell ELISA (Pierce Biotechnology) 4 h after treatment with $3 \mu \mathrm{M}$ (OciLy19) and $5 \mu \mathrm{M}$ (HT, HBL-1, U2932) Sph and Lt-Sph. Shown are means \pm $\mathrm{SD}$ of cleaved PARP normalized to $\alpha$-tubulin values, untreated control $=1.0, \mathrm{n}=3,{ }^{*} \mathrm{p}<0.05,{ }^{* * *} \mathrm{p}<0.001$. (D) Analysis of pJNK by ELISA (Abcam) $4 \mathrm{~h}$ after treatment with $3 \mu \mathrm{M}$ (OciLy19) and $5 \mu \mathrm{M}$ (HT, HBL-1, U2932) Sph and Lt-Sph. Shown are means \pm SD of pJNK values normalized to JNK values, untreated control = 1.0, $\mathrm{n}=3, * \mathrm{p}<0.05$.

The role of ceramide in Sph-induced apoptosis

Sph can either be phosphorylated to S1P, which binds and activates S1P receptors, or it can be acylated to ceramide, which also exerts pro-apoptotic activities using caspase dependent and -independent pathways. To test the relevance of ceramide generation for apoptosis induction after Sph treatment, DLBCL cell lines were treated with the ceramide synthase inhibitor fumonisin B1. The apoptotic rate of OciLy19, HT, and U2932 cell lines after treatment with camptothecin, Sph, and Lt-Sph was not significantly altered by addition of $25 \mu \mathrm{M}$ fumonisin B1, while HBL-1 cells showed a significant decrease of the apoptotic rate in Sph treated cells after fumonisin B1 treatment (Fig. 2A). The activity of fumonisin B1 was confirmed by LC/MS/MS quantification of the ceramide synthase substrate sphinganine in control and fumonisin B1 treated cells. Fumonisin B1 was shown to increase the amount of sphinganine via inhibition of ceramide synthases [47]. All DLBCL cell lines demonstrated a significant increase in sphinganine levels after treatment with fumonisin B1 (Fig. 2B). Increases were 2.5-fold and 10-fold for HT and OciLy19, and 32-fold and 39-fold for HBL1 and U2932 cells, respectively. Triple-quadrupole mass spectrometry revealed similar Sph and S1P accumulation with and without fumonisin B1 in all DLBCL cell lines (Fig. 2C). Intracellular Cer accumulation was only detectable in Sph treated HBL-1 and U2932, but not 


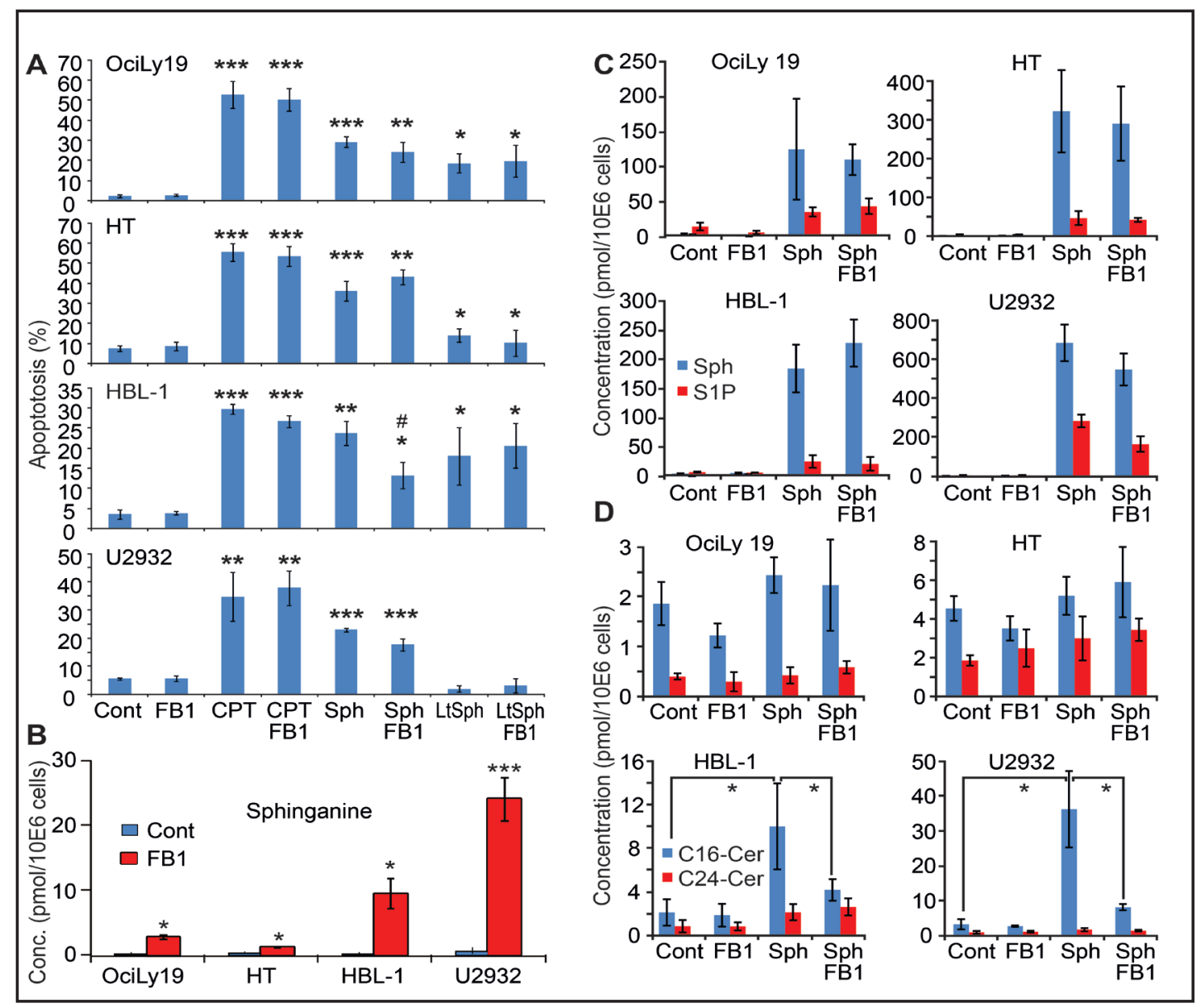

Fig. 2. The role of ceramide in Sph-induced apoptosis. (A) Apoptosis detected by FACS $4 \mathrm{~h}$ after treatment with $3 \mu \mathrm{M}$ (OciLy19) and $5 \mu \mathrm{M}$ (HT, HBL-1, U2932) Sph and Lt-Sph, and $20 \mu \mathrm{M}$ camptothecin (CPT) in the presence and absence of $25 \mu \mathrm{M}$ of the ceramide synthase inhibitor fumonisin B1 (FB1). Shown are means \pm SEM of 3 individual experiments representing the percentage of annexin V positive apoptotic cells, ${ }^{*} \mathrm{p}<0.05$, ${ }^{* *} \mathrm{p}<0.01,{ }^{* * *} \mathrm{p}<0.001$ compared to control, and $\# \mathrm{p}<0.05$ compared to corresponding sample without FB1 treatment. (B) LC/MS/MS analysis of the cellular content of sphinganine in DLBCL untreated and treated with $25 \mu \mathrm{M}$ fumonisin B1. Shown are means \pm SD of 3 individual experiments, ${ }^{*} \mathrm{p}<0.05,{ }^{* * *} \mathrm{p}<0.001$. (C, D) LC/MS/MS analysis of the cellular content of Sph and S1P (C) and C16-Cer and C24-Cer (D) in DLBCL untreated and treated with $3 \mu \mathrm{M}$ (OciLy19) and $5 \mu \mathrm{M}$ (HT, HBL-1, U2932) Sph in the presence and absence of $25 \mu \mathrm{M}$ fumonisin B1. Shown are means \pm SD of 3 individual experiments, ${ }^{*} \mathrm{p}<0.05$ for C16-Cer values.

in HT and OciLy19 DLBCL cell lines (Fig. 2D). It was efficiently blocked by $25 \mu \mathrm{M}$ fumonisin B1 (Fig. 2D). Thus, intracellular Cer accumulation was only evident in Sph-treated HBL-1 and U2932 DLBCL and did not account for most of the the observed apoptosis except for HBL-1 cells which showed significantly reduced apoptosis. OciLy19 and HT DLBCL did not show any alterations in ceramide levels nor in the apoptotic rate after ceramide synthase inhibition.

\section{Autophagy contributed to Sph-induced cell death}

In order to find out more about the underlying mechanisms involved in Sph-induced cell death, we looked for the development of acidic vesicular organelles 1 day after addition of Sph, Lt-Sph, and camptothecin. Acidic vesicular organelles are characteristic for autophagy and can be detected by FACS with acridin orange, which accumulates in acidic compartments and produces bright red fluorescence. All 4 tested DLBCL cell lines demonstrated an increase 


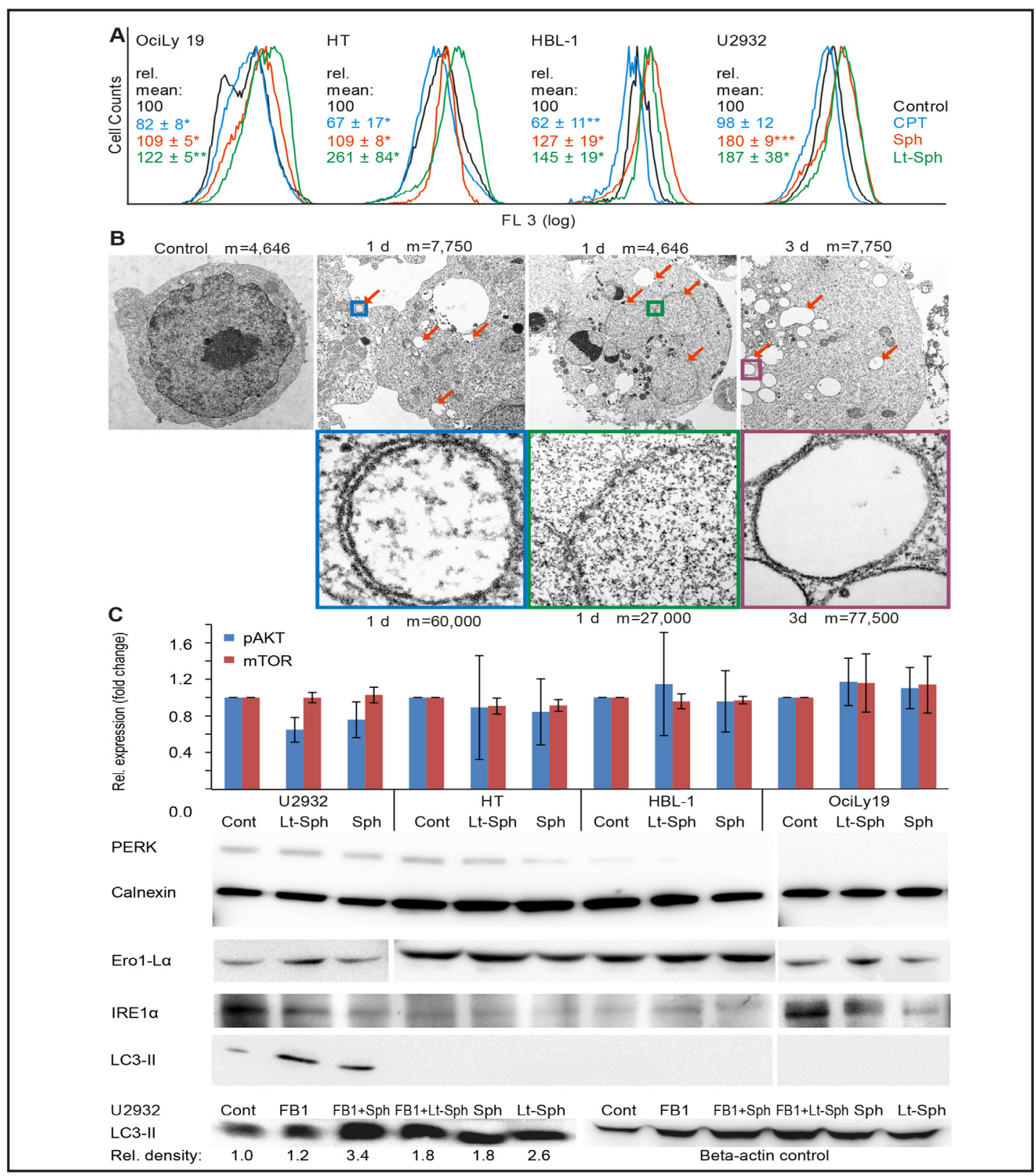

Fig. 3. Analysis of autophagy in DLBCL treated with $3 \mu \mathrm{M}$ (OciLy19) and $5 \mu \mathrm{M}$ (HT, HBL-1, U2932) Sph and Lt-Sph, and $10 \mu \mathrm{M}$ camptothecin. (A) FACS analysis of acidic vesicular organelles stained with acridine orange 1 day after treatment. Shown are histograms of 1 representative experiment out of 3 , and linear means \pm SD of 3 experiments, normalized to control $(=100),{ }^{*} p<0.05,{ }^{* *} p<0.01,{ }^{* * *} p<0.001$. (B) Electron microscopy pictures of untreated U2932 cells (control) and U2932 cells treated with $5 \mu$ M Sph for 1 and 3 days. Potential autophagic vesicles are marked with an arrow. Potential autophagosomal structures in colored squares are shown at higher magnification $(\mathrm{m})$ in equivalently colored frames to demonstrate the presence of double membranes characteristic for autophagosomes. (C) Western-blot analysis of the autophagic marker LC3-II and the ER stress markers PERK, calnexin, Ero1-L $\alpha$, and IRE1 $\alpha$ in combination with ELISA results of the phosphorylated signaling molecules pAKT and mTOR (pSer2448) $4 \mathrm{~h}$ after treatment with 3 $\mu \mathrm{M}$ (OciLy19) and $5 \mu \mathrm{M}$ (HT, HBL-1, U2932) Sph and Lt-Sph with and without prior addition of $25 \mu \mathrm{M}$ fumonisin B1 (FB1). Shown are representative Western-blot results from 2-3 independent experiments and means \pm SD of ELISA results for AKT and mTOR phosphorylation, untreated control =1.0, $\mathrm{n}=3$. pAKT values were normalized to AKT values, the relative density of LC3-II Western blot signals was normalized to the respective beta-actin expression. 
Fig. 4. Influence of Sph and Lt-Sph on PKC activity and IL-10 production in DLBCL cell lines. (A) Measurement of PKC activity in the presence and absence of indicated molar ratios of PS/Sph and PS/ Lt-Sph. Shown are means \pm SEM, $n=2,{ }^{*} \mathrm{p}<0.05$. (B) Measurement of IL-10 in the supernatant of HBL1 and U2932 DLBCL cell lines after treatment with the indicated $\mu \mathrm{M}$ concentrations of Sph and Lt-Sph. Shown are means \pm SD, $n=3, * * * p<0.001$.

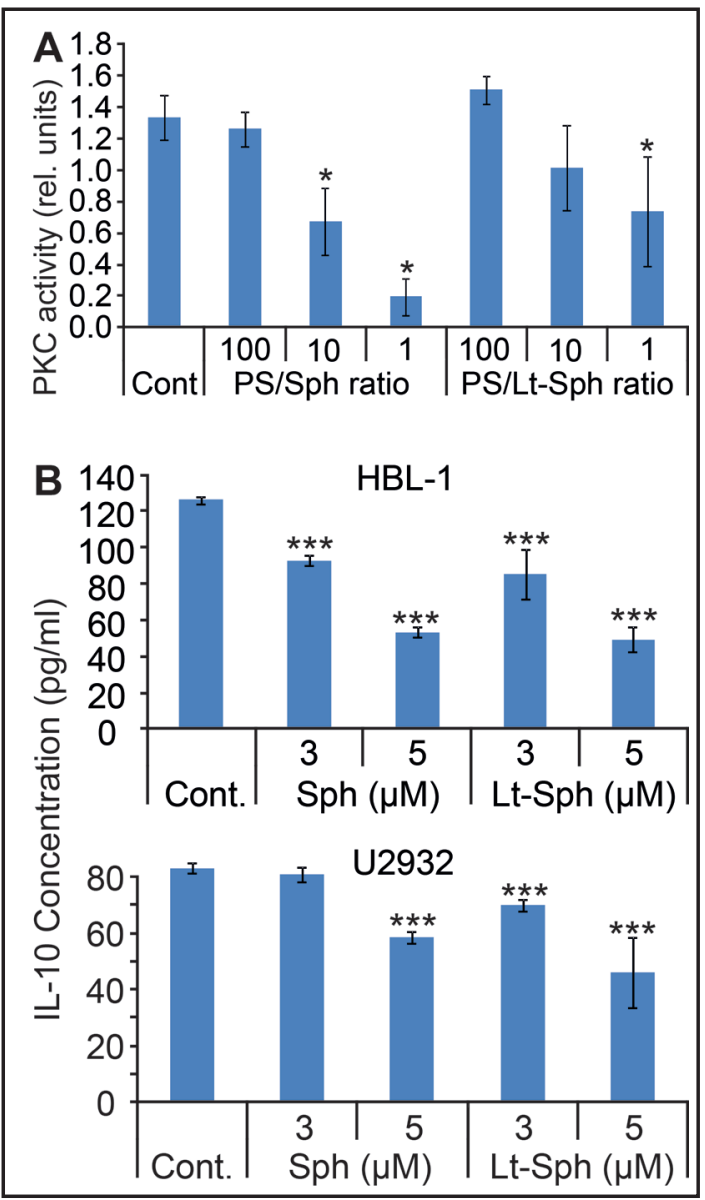

of red fluorescence as determined by FACS after treatment with Sph and Lt-Sph, but not with camptothecin (Fig. 3A). While HBL-1 and U2932 DLBCL cell lines responded equally well to both stimuli, OciLy19 and HT revealed a greater shift in the red fluorescence after Lt-Sph stimulation than after Sph-stimulation. U2932 cells did not respond to Lt-Sph treatment with apoptosis (Fig. 1B, 2A), but they showed a similar increase in acidic compartments compared to Sph treatment (Fig. 3A). Electron microscopy of U2932 cells 1 and 3 days after treatment with Sph uncovered the presence of potential autophagosomes or secondary lysosomes, respectively. In comparison to control cells, Sph-treated cells developed increased vacuole formation one and three days after Sph addition (Fig. 3B). Examination of these vacuoles at higher magnification revealed the presence of double-membranes which are characteristic for autophagosomes (Fig. 3B). The presence of these double-membrane vesicles together with the observed increase in acidic compartments are in support for the additional induction of autophagy after treatment with Sph and Lt-Sph (Fig. 3A-B). Further evidence derived from the analysis of the autophagy marker light chain 3 (LC3), which is cleaved at the carboxy terminus to the LC3-I form immediately after its synthesis. LC3-I is converted to LC3-II via lipidation and becomes associated with autophagic vesicles. The conversion of LC3 to the lower migrating form LC3-II is indicative for ongoing autophagy in Western blot analyses [48]. Treatment of DLBCL cell lines with Sph and Lt-Sph led to an increase of LC3-II in U2932 DLBCL, while no specific signal was observed in OciLy19, HT, and HBL-1 DLBCL cell lines (Fig. 3C). LC3-II formation was not inhibited by fumonisin B1 (Fig. 3C). Neither the protein kinase AKT nor its downstream target molecule and suppressor of autophagy mTOR were influenced by Sph or Lt-Sph treatment, indicating the dispensability of the AKT/mTor signalling pathway in this context (Fig. 3C). Further analysis of markers for endoplasmic reticulum (ER) stress signalling including PKR-like ER kinase (PERK), calnexin, endoplasmic oxidoreducin-1 (Ero1), and the inositol-requiring enzyme 1 (IRE1) also eliminated a 


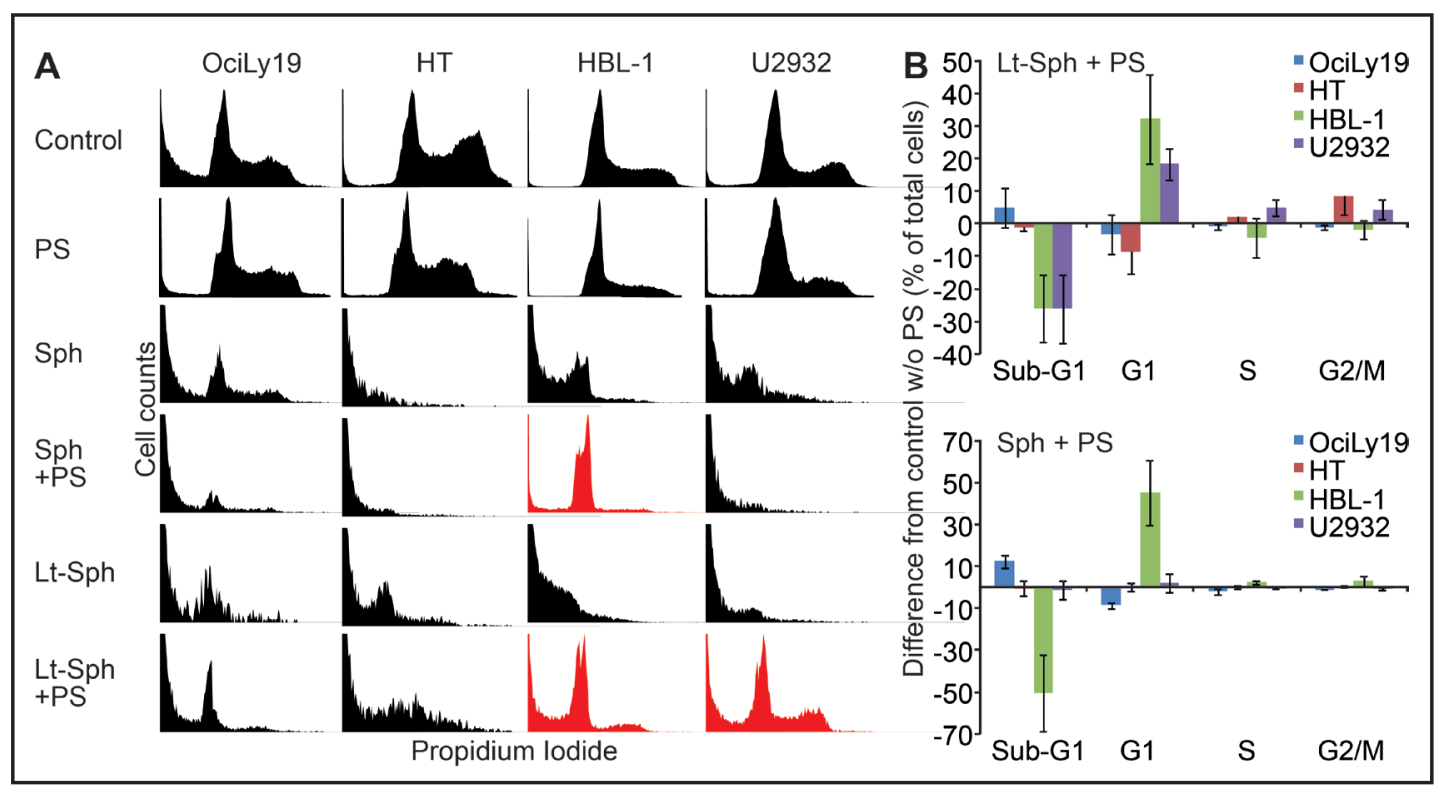

Fig. 5. Competitive effect of the PKC activator PS on Sph and Lt-Sph induced cell death in ABC DLBCL. (A) Cell cycle analysis of DLBCL cell lines treated for 3 days with $3 \mu \mathrm{M}$ (OciLy19) and $5 \mu \mathrm{M}$ (HT, HBL-1, U2932) Sph and Lt-Sph in the presence and absence of $15 \mu$ M PS. Shown are histograms of 1 representative experiment out of 3. (B) Quantification of cell populations in different cell cycle stages 3 days after treatment with $3 \mu \mathrm{M}$ (OciLy19) and $5 \mu \mathrm{M}$ (HT, HBL-1, U2932) Sph and Lt-Sph in the presence and absence of $15 \mu \mathrm{M}$ PS. Bars represent differences between cell populations with and without PS co-treatment. Shown are means \pm SEM, $n=2-3$.

pertinent role of ER stress-related pathways for induction of the observed cell death (Fig. 3C). Thus, U2932 DLBCL responded to Sph and Lt-Sph treatment with increased autophagyrelated LC3-II generation, while no detectable changes of this protein were observed with OciLy19, HT, and HBL-1 DLBCL cell lines.

\section{PKC inhibition by Sph and Lt-Sph}

The observed differences of DLBCL cell lines to respond to Sph and Lt-Sph persuaded us of investigating PKC signalling as a third potential contributor to Sph and Lt-Sph induced cell death. Recent findings indicated that PKC signalling is an important survival factor of $A B C$, but not GCB DLBCL [4]. In vitro PKC activity assays demonstrated that both Sph and Lt-Sph inhibited PKC in a concentration-dependent manner, with Sph being more effective than LtSph in competing with the PKC activator PS for binding to the C1 domain (Fig. 4A). Since PKC signalling was shown to induce the production of the downstream effector cytokine IL-10, which promotes proliferation and survival of B cells, quantification of IL-10 was performed by ELISA. While the DLBCL cell lines OciLy19 and HT hardly produced any IL-10 (data not shown), the DLBCL cell lines HBL-1 and U2932 produced IL-10 at physiologically relevant levels. Addition of Sph and Lt-Sph reduced the amount of secreted IL-10 within 4 hours (Fig. 4B). The effect was similar with Sph and Lt-Sph, and HBL-1 cells were more sensitive than U2932 cells in this assay. PKC activity was therefore compromised by Sph and Lt-Sph treatment, and addition of Sph and Lt-Sph reduced the amount of IL-10 release in the IL-10 expressing DLBCL cell lines HBL-1 and U2932.

The PKC activator PS partially rescued HBL-1 and U2932 DLBCL from cell death

To test the relevance of PKC inhibition for Sph and Lt-Sph induced cell death, OciLy19, HT, HBL-1, and U2932 DLBCL cell lines were incubated with $15 \mu \mathrm{M}$ of the PKC activator PS in order to compete with the PKC inhibitors Sph and Lt-Sph for binding to the C1 domain [22]. As part of the regulatory domain or the amino-terminus of the PKCs, the C1 domain 
Table 1. Summary of effects of Sph and Lt-Sph on investigated DLBCL cell lines. * Acridine orange staining only; \# IL-10 production only; + strong response; o weak response; - no response

\begin{tabular}{lcccccccc}
\hline & \multicolumn{2}{c}{ OciLy19 } & \multicolumn{2}{c}{ HT } & \multicolumn{2}{c}{ HBL-1 } & \multicolumn{2}{c}{ U2932 } \\
& Sph & Lt-Sph & Sph & Lt-Sph & Sph & Lt-Sph & Sph & Lt-Sph \\
\hline Cell growth block & + & + & + & 0 & + & + & + & + \\
Apoptosis & + & + & + & 0 & + & + & + & - \\
pJNK \& cleaved PARP & + & - & - & - & - & - & - & - \\
Autophagy & $\mathrm{O}^{*}$ & $\mathrm{O}^{*}$ & $\mathrm{O}^{*}$ & $+^{*}$ & $+^{*}$ & $+^{*}$ & + & + \\
pAkt \& mTOR & - & - & - & - & - & - & - & - \\
ER stress & - & - & - & - & - & - & - & - \\
PKC inhibition & - & - & - & - & + & + & $\mathrm{O}^{\#}$ & 0 \\
\hline
\end{tabular}

is present in all PKC isoforms. PS was ineffective on OciLy19 and HT DLBCL cell lines, but HBL-1 and U2932 were partially rescued from Lt-Sph induced cell death in the presence of $15 \mu$ M PS. A significant amount of HBL-1 and U2932 cells remained in the G1 phase of the cell cycle 3 days after co-treatment with $5 \mu \mathrm{M}$ Lt-Sph and $15 \mu \mathrm{M}$ PS, while the same cells treated with Lt-Sph alone were mostly shifted to the sub-G1 phase (Fig. 5A). PS similarly rescued HBL-1, but not U2932 cells from cell death during co-treatment with $5 \mu \mathrm{M} \mathrm{Sph}$, again indicating the activity of additional pathways leading to cell death in U2932 cells after Sph treatment. Quantitative analysis of several different experiments demonstrated $20-30 \%$ decrease of HBL-1 and U2932 cells in the sub-G1 phase and a similar increase of both DLBCL cell lines in the G1 phase after co-treatment with $5 \mu \mathrm{M}$ Lt-Sph and $15 \mu \mathrm{M}$ PS compared to single treatment with $5 \mu \mathrm{M}$ Lt-Sph alone (Fig. 5B). No significant changes were observed with OciLy19 and HT DLBCL cell lines. Sph-induced cell death was prevented by PS only in HBL-1 cells, with 40-50\% less cells in the sub-G1 phase and equivalently more cells in the G1 phase.

\section{Discussion}

Sphingolipids like S1P, Sph, and ceramide have long been implicated as regulators of cell fate $[7,8]$. Ceramide was suggested as a target molecule to combat drug resistance of tumors [49], and an anti-S1P antibody is currently a promising candidate for treatment of solid tumors [50]. Cell culture experiments with DLBCL cell lines that were incubated with Sph and Lt-Sph manifested significant inhibition of cell growth (Fig. 1A), while incubation of cells with similar concentrations of S1P was ineffective (data not shown). These results suggest that Sph accumulation could be an alternative treatment option for DLBCL. To this end we investigated several different signaling pathways that are potentially involved in Sphand Lt-Sph-induced cell death (Table 1).

Apoptosis induced by Sph has been described before [23, 24, 29], but the physiological relevance remained unclear. The Sph analog FTY720 also induces apoptosis, but only at concentrations similar to Sph, which are never reached during treatment [51]. But how could relevant Sph concentrations been attained in vivo? One possible way would be the pharmacological inhibition of the S1P-lyase. Sph concentrations reach levels after S1P-lyase inhibition up to $100 \mu \mathrm{mol} / \mathrm{kg}$ tissue weight in mice, which would be sufficient for the described cell death inducing effects in aggressive lymphomas [33]. LX2931 is an S1P-lyase inhibitor that passed phase 2a clinical studies for treatment of the autoimmune disease rheumatoid arthritis [52]. Our results suggest that Sph accumulation by S1P-lyase inhibition is a so far unappreciated effect that may be beneficial for DLBCL treatment. On the other hand S1P-lyase inhibition results in an even more dramatic increase of S1P, and S1P was shown to activate pro-survival signaling pathways in cells. Some of these effects were attributed to S1PR1 signaling [9], while others may be induced by additional S1PRs [27] or by intracellular targets of S1P [53]. These pro-survival activities of S1P may therefore compensate for the lethal effects of Sph. It should be noted however that excess amounts of 
S1P result in prolonged internalization and functional inhibition of S1PR1 [33], which may also disrupt pro-survival signaling of S1P, at least partially.

Genetic profiling of the cell lines used indicated that OciLy19 and HT belong to the group of GCB-DLBCL, while HBL-1 and U2932 share main characteristics with ABC-DLBCL $[35,54]$. This likely explains the different PKC-mediated responses in OciLy19 and HT versus HBL-1 and U2932 DLBCL cell lines since survival of ABC-DLBCL is much more reliant on constitutive PKC signaling than GCB-DLBCL. Intriguingly HBL-1 and U2932 cell lines also produced more Cer than OciLy19 and HT upon incubation with Sph, and Cer production contributed to Sph-induced apoptosis in HBL-1 cells (Fig. 2A). Several reports indicate that Cer activates PKC $[16,55]$, which may contribute to constitutive PKC signaling in ABC-DLBCL. Sph itself was able to interfere with PKC signaling (Fig. 4A) and counteracted pro-survival signals of PKC as a competitive inhibitor of the PKC activator PS. Lt-Sph also inhibited PKC in vitro, but with lower efficacy (Fig. 4A). Lt-Sph was more stable than Sph in cell culture (data not shown), which may explain its similar efficacy as competitive PKC inhibitor in HBL-1 cells and even better efficacy in U2932 cells compared to Sph.

Besides accumulation of endogenous Sph by S1P-lyase inhibition, SphK inhibitors may also be used for DLBCL treatment due to their structural similarities to Sph [34]. Potential effects of those inhibitors certainly need to be tested individually. This study provides the basis to specifically look at apoptosis, PKC-inhibition, and autophagy in DLBCL. Since S1P is typically considered as a pro-survival factor [7], increased cell death observed after treatment with inhibitors of the S1P-producing SphKs are usually assigned to the presence of lower S1P levels. This study however demonstrates that accumulation of the SphK substrate Sph can significantly impair DLBCL growth and survival, suggesting that SphK inhibiting analogues of Sph may be similarly effective. Agents enhancing endogenous cellular Sph levels like LX2931 [52] and Sph analogues like Safingol [56] may achieve their activity not only by altering S1P levels and signaling, but also by inducing apoptosis, PKC-inhibition, and autophagy as shown in this study. Future drug candidates in the sphingolipid field for cancer treatment may therefore shift from inhibitors of S1P signaling to non-phosphorylatable Sph analogues. An autonomous phosphorylation independent inhibitory activity of Sph and FTY720 was also demonstrated for the TRPM7 channel [57], which further supports specific cellular activity of Sph.

Sph induced cell death and blocked proliferation by various means, including apoptosis, autophagy, and PKC inhibition. This variability of cellular functions seems to be important for the overall efficacy of Sph to prevent lymphoma cell growth. Despite striking differences in the efficacy of Sph and Lt-Sph on different DLBCL, the final impact on cell growth and mortality was similar due to the combined activity of different cellular pathways. The broadband impact of Sph across different types of DLBCL introduces sphingosine as a cytostatic drug for lymphoma treatment. Current targeting strategies include the disruption of cancer-specific cellular functions like PKC signalling in ABC DLBCL [35]. Sphingosine accumulation e.g. by S1P-lyase inhibition or by application of Sph analogues provides a different strategy which could be an interesting alternative for combination therapy.

\section{Disclosure Statement}

The authors declare no conflict of interests.

\section{Acknowledgements}

The authors thank Georg Lenz for kindly providing the DLBCL cell lines, Petra Schrade for electron microscopy preparations, and Clemens Schmitt for helpful discussions and comments. This work was supported by the Deutsche Forschungsgemeinschaft (DFG), 
grant GR 1943/3-1 (to M. H. G.), and the priority program 1267 "sphingolipids - signals and disease", grant GR 1943/2-2 (to M. H. G.).

\section{References}

1 American Cancer Society. Cancer Facts \& Figures 2013. Atlanta: American Cancer Society; 2013.

2 Staudt LM, Dave S: The biology of human lymphoid malignancies revealed by gene expression profiling. Adv Immunol 2005;87:163-208.

-3 Alizadeh AA, Staudt LM: Genomic-scale gene expression profiling of normal and malignant immune cells. Curr Opin Immunol 2000;12:219-225.

4 Davis RE, Ngo VN, Lenz G, Tolar P, Young RM, Romesser PB, Kohlhammer H, Lamy L, Zhao H, Yang Y, Xu W, Shaffer AL, Wright G, Xiao W, Powell J, Jiang JK, Thomas CJ, Rosenwald A, Ott G, Muller-Hermelink HK, Gascoyne RD, Connors JM, Johnson NA, Rimsza LM, Campo E, Jaffe ES, Wilson WH, Delabie J, Smeland EB, Fisher RI, Braziel RM, Tubbs RR, Cook JR, Weisenburger DD, Chan WC, Pierce SK, Staudt LM: Chronic active b-cell-receptor signalling in diffuse large b-cell lymphoma. Nature 2010;463:88-92.

5 Pfeifer M, Grau M, Lenze D, Wenzel SS, Wolf A, Wollert-Wulf B, Dietze K, Nogai H, Storek B, Madle H, Dorken B, Janz M, Dirnhofer S, Lenz P, Hummel M, Tzankov A, Lenz G: Pten loss defines a pi3k/akt pathwaydependent germinal center subtype of diffuse large b-cell lymphoma. Proc Natl Acad Sci U S A 2013

6 Lenz G, Wright G, Dave SS, Xiao W, Powell J, Zhao H, Xu W, Tan B, Goldschmidt N, Iqbal J, Vose J, Bast M, Fu K, Weisenburger DD, Greiner TC, Armitage JO, Kyle A, May L, Gascoyne RD, Connors JM, Troen G, Holte H, Kvaloy S, Dierickx D, Verhoef G, Delabie J, Smeland EB, Jares P, Martinez A, Lopez-Guillermo A, Montserrat E, Campo E, Braziel RM, Miller TP, Rimsza LM, Cook JR, Pohlman B, Sweetenham J, Tubbs RR, Fisher RI, Hartmann E, Rosenwald A, Ott G, Muller-Hermelink HK, Wrench D, Lister TA, Jaffe ES, Wilson WH, Chan WC, Staudt LM, Lymphoma/Leukemia Molecular Profiling P: Stromal gene signatures in large-b-cell lymphomas. N Engl J Med 2008;359:2313-2323.

7 Cuvillier O, Pirianov G, Kleuser B, Vanek PG, Coso OA, Gutkind S, Spiegel S: Suppression of ceramidemediated programmed cell death by sphingosine-1-phosphate. Nature 1996;381:800-803.

$>8$ Sweeney EA, Sakakura C, Shirahama T, Masamune A, Ohta H, Hakomori S, Igarashi Y: Sphingosine and its methylated derivative $n, n$-dimethylsphingosine (dms) induce apoptosis in a variety of human cancer cell lines. Int J Cancer 1996;66:358-366.

-9 Lee H, Deng J, Kujawski M, Yang C, Liu Y, Herrmann A, Kortylewski M, Horne D, Somlo G, Forman S, Jove $\mathrm{R}$, Yu H: Stat3-induced s1pr1 expression is crucial for persistent stat3 activation in tumors. Nat Med 2010;16:1421-1428.

10 Morad SA, Cabot MC: Ceramide-orchestrated signalling in cancer cells. Nat Rev Cancer 2013;13:51-65.

11 Young MM, Kester M, Wang HG: Sphingolipids: Regulators of crosstalk between apoptosis and autophagy. J Lipid Res 2013;54:5-19.

12 Siskind LJ, Colombini M: The lipids c2- and c16-ceramide form large stable channels. Implications for apoptosis. J Biol Chem 2000;275:38640-38644.

-13 Siskind LJ, Kolesnick RN, Colombini M: Ceramide channels increase the permeability of the mitochondrial outer membrane to small proteins. J Biol Chem 2002;277:26796-26803.

14 Chalfant CE, Rathman K, Pinkerman RL, Wood RE, Obeid LM, Ogretmen B, Hannun YA: De novo ceramide regulates the alternative splicing of caspase 9 and bcl-x in a549 lung adenocarcinoma cells. Dependence on protein phosphatase-1. J Biol Chem 2002;277:12587-12595.

$>15$ Heinrich M, Wickel M, Schneider-Brachert W, Sandberg C, Gahr J, Schwandner R, Weber T, Saftig P, Peters C, Brunner J, Krönke M, Schütze S: Cathepsin d targeted by acid sphingomyelinase-derived ceramide. Embo J 1999;18:5252-5263.

16 Wang YM, Seibenhener ML, Vandenplas ML, Wooten MW: Atypical pkc zeta is activated by ceramide, resulting in coactivation of nf-kappab/jnk kinase and cell survival. J Neurosci Res 1999;55:293-302.

17 Spassieva SD, Mullen TD, Townsend DM, Obeid LM: Disruption of ceramide synthesis by cers2 downregulation leads to autophagy and the unfolded protein response. Biochem J 2009;424:273-283.

18 Zhou H, Summers SA, Birnbaum MJ, Pittman RN: Inhibition of akt kinase by cell-permeable ceramide and its implications for ceramide-induced apoptosis. J Biol Chem 1998;273:16568-16575. 
19 Westwick JK, Bielawska AE, Dbaibo G, Hannun YA, Brenner DA: Ceramide activates the stress-activated protein kinases. J Biol Chem 1995;270:22689-22692.

20 Scarlatti F, Bauvy C, Ventruti A, Sala G, Cluzeaud F, Vandewalle A, Ghidoni R, Codogno P: Ceramidemediated macroautophagy involves inhibition of protein kinase $b$ and up-regulation of beclin 1. J Biol Chem 2004;279:18384-18391.

21 Daido S, Kanzawa T, Yamamoto A, Takeuchi H, Kondo Y, Kondo S: Pivotal role of the cell death factor bnip3 in ceramide-induced autophagic cell death in malignant glioma cells. Cancer Res 2004;64:4286-4293.

22 Hannun YA, Loomis CR, Merrill AH, Jr., Bell RM: Sphingosine inhibition of protein kinase c activity and of phorbol dibutyrate binding in vitro and in human platelets. J Biol Chem 1986;261:12604-12609.

-23 Jarvis WD, Fornari FA, Jr., Auer KL, Freemerman AJ, Szabo E, Birrer MJ, Johnson CR, Barbour SE, Dent P, Grant S: Coordinate regulation of stress- and mitogen-activated protein kinases in the apoptotic actions of ceramide and sphingosine. Mol Pharmacol 1997;52:935-947.

24 Cuvillier O, Nava VE, Murthy SK, Edsall LC, Levade T, Milstien S, Spiegel S: Sphingosine generation, cytochrome c release, and activation of caspase-7 in doxorubicin-induced apoptosis of mcf7 breast adenocarcinoma cells. Cell Death Differ 2001;8:162-171.

25 Liu H, Sugiura M, Nava VE, Edsall LC, Kono K, Poulton S, Milstien S, Kohama T, Spiegel S: Molecular cloning and functional characterization of a novel mammalian sphingosine kinase type 2 isoform. J Biol Chem 2000;275:19513-19520.

-26 Olivera A, Kohama T, Tu Z, Milstien S, Spiegel S: Purification and characterization of rat kidney sphingosine kinase. J Biol Chem 1998;273:12576-12583.

27 Mihovilovic M, Robinette JB, DeKroon RM, Sullivan PM, Strittmatter WJ: High-fat/high-cholesterol diet promotes a s1p receptor-mediated antiapoptotic activity for vldl. J Lipid Res 2007;48:806-815.

28 Colie S, Van Veldhoven PP, Kedjouar B, Bedia C, Albinet V, Sorli SC, Garcia V, Djavaheri-Mergny M, Bauvy C, Codogno P, Levade T, Andrieu-Abadie N: Disruption of sphingosine 1-phosphate lyase confers resistance to chemotherapy and promotes oncogenesis through bcl-2/bcl-xl upregulation. Cancer Res 2009;69:93469353.

29 Sakakura C, Sweeney EA, Shirahama T, Hakomori S, Igarashi Y: Suppression of bcl-2 gene expression by sphingosine in the apoptosis of human leukemic hl-60 cells during phorbol ester-induced terminal differentiation. FEBS Lett 1996;379:177-180.

30 Pyne NJ, Pyne S: Sphingosine 1-phosphate and cancer. Nat Rev Cancer 2010;10:489-503.

31 Shida D, Takabe K, Kapitonov D, Milstien S, Spiegel S: Targeting sphk1 as a new strategy against cancer. Curr Drug Targets 2008;9:662-673.

32 Sensken SC, Bode C, Nagarajan M, Peest U, Pabst O, Gräler MH: Redistribution of sphingosine 1-phosphate by sphingosine kinase 2 contributes to lymphopenia. J Immunol 2010;184:4133-4142.

-33 Weber C, Krueger A, Münk A, Bode C, Van Veldhoven PP, Gräler MH: Discontinued postnatal thymocyte development in sphingosine 1-phosphate-lyase-deficient mice. J Immunol 2009;183:4292-4301.

34 Wong L, Tan SS, Lam Y, Melendez AJ: Synthesis and evaluation of sphingosine analogues as inhibitors of sphingosine kinases. J Med Chem 2009;52:3618-3626.

35 Naylor TL, Tang H, Ratsch BA, Enns A, Loo A, Chen L, Lenz P, Waters NJ, Schuler W, Dörken B, Yao YM, Warmuth M, Lenz G, Stegmeier F: Protein kinase c inhibitor sotrastaurin selectively inhibits the growth of cd79 mutant diffuse large b-cell lymphomas. Cancer Res 2011;71:2643-2653.

-36 Andréani P, Gräler MH: Comparative quantification of sphingolipids and analogs in biological samples by high-performance liquid chromatography after chloroform extraction. Anal Biochem 2006;358:239-246.

-37 Bode C, Gräler MH: Quantification of sphingosine-1-phosphate and related sphingolipids by liquid chromatography coupled to tandem mass spectrometry. Methods Mol Biol 2012;874:33-44.

-38 Richardson KC, Jarett L, Finke EH: Embedding in epoxy resins for ultrathin sectioning in electron microscopy. Stain Technol 1960;35:313-323.

39 Reynolds ES: The use of lead citrate at high ph as an electron-opaque stain in electron microscopy. J Cell Biol 1963;17:208-212.

40 Hauser JM, Buehrer BM, Bell RM: Role of ceramide in mitogenesis induced by exogenous sphingoid bases. J Biol Chem 1994;269:6803-6809.

41 Nakamura H, Takashiro Y, Hirabayashi T, Horie S, Koide Y, Nishida A, Murayama T: Effects of synthetic sphingosine-1-phosphate analogs on arachidonic acid metabolism and cell death. Biochem Pharmacol 2004;68:2187-2196. 
42 Olivera A, Zhang H, Carlson RO, Mattie ME, Schmidt RR, Spiegel S: Stereospecificity of sphingosine-induced intracellular calcium mobilization and cellular proliferation. J Biol Chem 1994;269:17924-17930.

43 Liu LF, Desai SD, Li TK, Mao Y, Sun M, Sim SP: Mechanism of action of camptothecin. Ann N Y Acad Sci 2000;922:1-10.

44 Hengartner MO: The biochemistry of apoptosis. Nature 2000;407:770-776.

45 Bae S, Yalamarti B, Kang PM: Role of caspase-independent apoptosis in cardiovascular diseases. Front Biosci 2008;13:2495-2503.

-46 Simbulan-Rosenthal CM, Rosenthal DS, Iyer S, Boulares H, Smulson ME: Involvement of parp and poly(adpribosyl)ation in the early stages of apoptosis and DNA replication. Mol Cell Biochem 1999;193:137-148.

47 Wang E, Norred WP, Bacon CW, Riley RT, Merrill AH, Jr.: Inhibition of sphingolipid biosynthesis by fumonisins. Implications for diseases associated with fusarium moniliforme. J Biol Chem 1991;266:1448614490.

48 Tanida I, Ueno T, Kominami E: Lc3 and autophagy. Methods Mol Biol 2008;445:77-88.

49 Lucci A, Giuliano AE, Han TY, Dinur T, Liu YY, Senchenkov A, Cabot MC: Ceramide toxicity and metabolism differ in wild-type and multidrug-resistant cancer cells. Int J Oncol 1999;15:535-540.

-50 Visentin B, Vekich JA, Sibbald BJ, Cavalli AL, Moreno KM, Matteo RG, Garland WA, Lu Y, Yu S, Hall HS, Kundra V, Mills GB, Sabbadini RA: Validation of an anti-sphingosine-1-phosphate antibody as a potential therapeutic in reducing growth, invasion, and angiogenesis in multiple tumor lineages. Cancer Cell 2006;9:225-238.

51 Brinkmann V, Wilt C, Kristofic C, Nikolova Z, Hof RP, Chen S, Albert R, Cottens S: Fty720: Dissection of membrane receptor-operated, stereospecific effects on cell migration from receptor-independent antiproliferative and apoptotic effects. Transplant Proc 2001;33:3078-3080.

52 Bagdanoff JT, Donoviel MS, Nouraldeen A, Carlsen M, Jessop TC, Tarver J, Aleem S, Dong L, Zhang H, Boteju L, Hazelwood J, Yan J, Bednarz M, Layek S, Owusu IB, Gopinathan S, Moran L, Lai Z, Kramer J, Kimball SD, Yalamanchili P, Heydorn WE, Frazier KS, Brooks B, Brown P, Wilson A, Sonnenburg WK, Main A, Carson KG, Oravecz T, Augeri DJ: Inhibition of sphingosine 1-phosphate lyase for the treatment of rheumatoid arthritis: Discovery of (e)-1-(4-((1r,2s,3r)-1,2,3,4-tetrahydroxybutyl)-1h-imidazol-2-yl)ethanone oxime (lx2931) and (1r,2s,3r)-1-(2-(isoxazol-3-yl)-1h-imidazol-4-yl)butane-1,2,3,4-tetraol (lx2932). J Med Chem 2010;53:8650-8662.

53 Alvarez SE, Harikumar KB, Hait NC, Allegood J, Strub GM, Kim EY, Maceyka M, Jiang H, Luo C, Kordula T, Milstien S, Spiegel S: Sphingosine-1-phosphate is a missing cofactor for the e3 ubiquitin ligase traf2. Nature 2010;465:1084-1088.

54 Alizadeh AA, Eisen MB, Davis RE, Ma C, Lossos IS, Rosenwald A, Boldrick JC, Sabet H, Tran T, Yu X, Powell JI, Yang L, Marti GE, Moore T, Hudson J Jr, Lu L, Lewis DB, Tibshirani R, Sherlock G, Chan WC, Greiner TC, Weisenburger DD, Armitage JO, Warnke R, Levy R, Wilson W, Grever MR, Byrd JC, Botstein D, Brown PO, Staudt LM: Distinct types of diffuse large b-cell lymphoma identified by gene expression profiling. Nature 2000;403:503-511.

55 Fox TE, Houck KL, O’Neill SM, Nagarajan M, Stover TC, Pomianowski PT, Unal O, Yun JK, Naides SJ, Kester M: Ceramide recruits and activates protein kinase c zeta (pkc zeta) within structured membrane microdomains. J Biol Chem 2007;282:12450-12457.

-56 Dickson MA, Carvajal RD, Merrill AH Jr, Gonen M, Cane LM, Schwartz GK: A phase i clinical trial of safingol in combination with cisplatin in advanced solid tumors. Clin Cancer Res 2011;17:2484-2492.

57 Qin X, Yue Z, Sun B, Yang W, Xie J, Ni E, Feng Y, Mahmood R, Zhang Y, Yue L: Sphingosine and fty720 are potent inhibitors of the transient receptor potential melastatin 7 (trpm7) channels. Br J Pharmacol 2013;168:1294-1312. 